\title{
Preparing Alumina-Supported Gold Nanowires for Alcohol
}

\section{Oxidation}

Yoshiro Imura, ${ }^{1,{ }^{*}}$ Motoki Maniwa, ${ }^{1}$ Kazuki Iida, ${ }^{1}$ Haruna Saito, ${ }^{1}$ Clara Morita-Imura, ${ }^{2}$ and Takeshi Kawai ${ }^{1, *}$

${ }^{1}$ Department of Industrial Chemistry, Tokyo University of Science, 1-3 Kagurazaka, Shinjuku-ku, Tokyo 162-8601, Japan.

${ }^{2}$ Department of Chemistry, Faculty of Science, Ochanomizu University, 2-1-1 Otsuka, Bunkyo-ku, Tokyo 112-8610, Japan. 


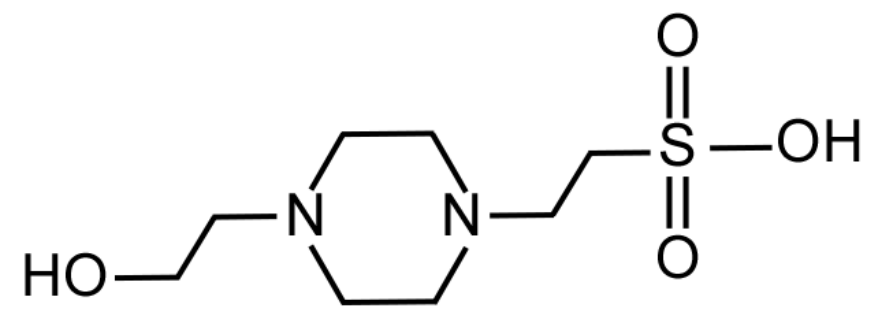

Figure S1. Molecular structure of HEPES.

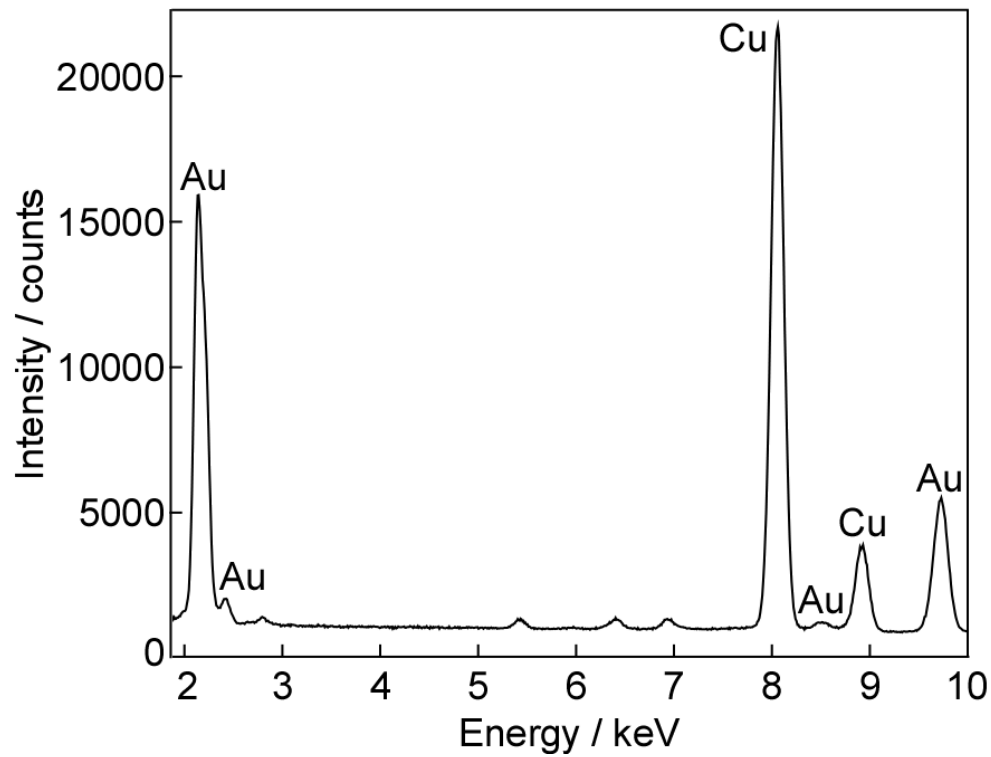

Figure S2. TEM-EDX spectrum of AuNWs. The $\mathrm{Cu}$ peaks arise from the TEM $\mathrm{Cu}$ grid. 
(a)

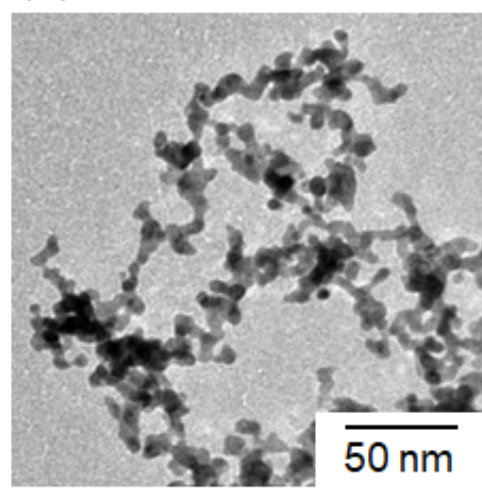

(b)

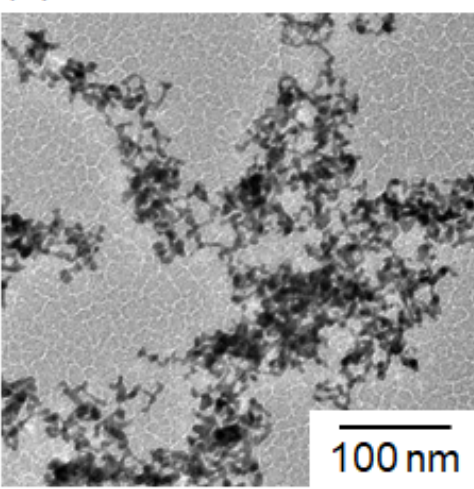

(c)

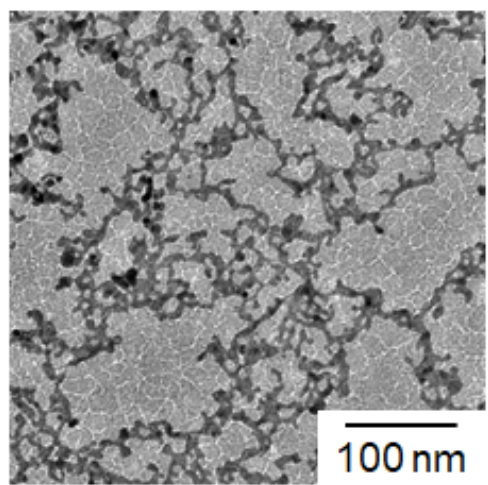

Figure S3. TEM images of AuNWs prepared using (a) 50, (b) 100, and (c) $200 \mathrm{mM}$ HEPES aqueous solution.

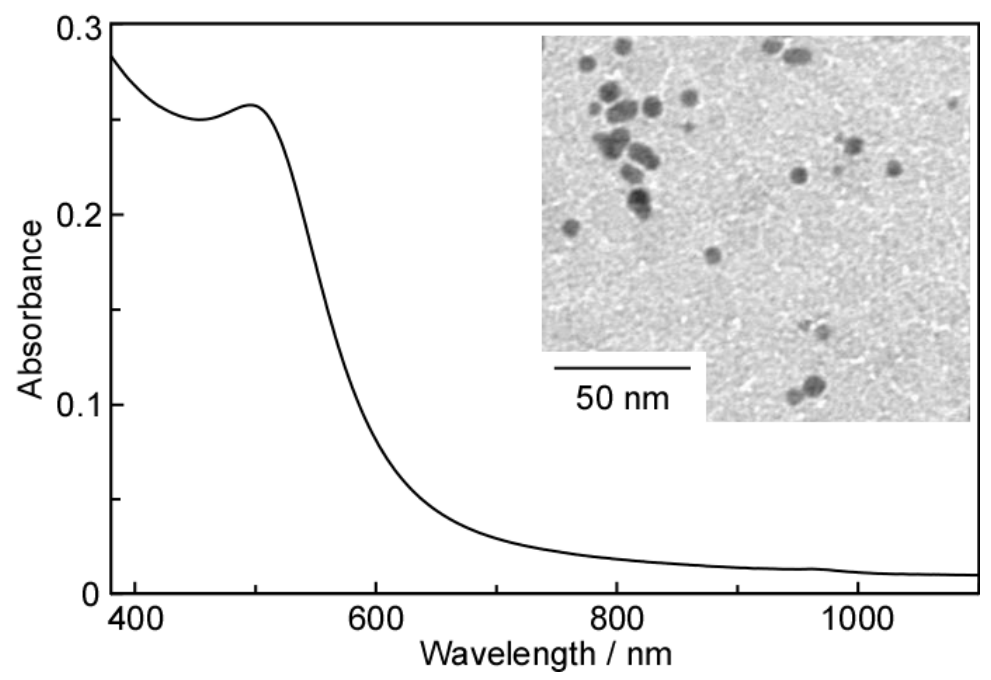

Figure S4. UV-vis spectrum and TEM image of Au nanocrystals. The concentrations of HEPES and $\mathrm{NaBH}_{4}$ are 15 and $24 \mathrm{mM}$, respectively. 


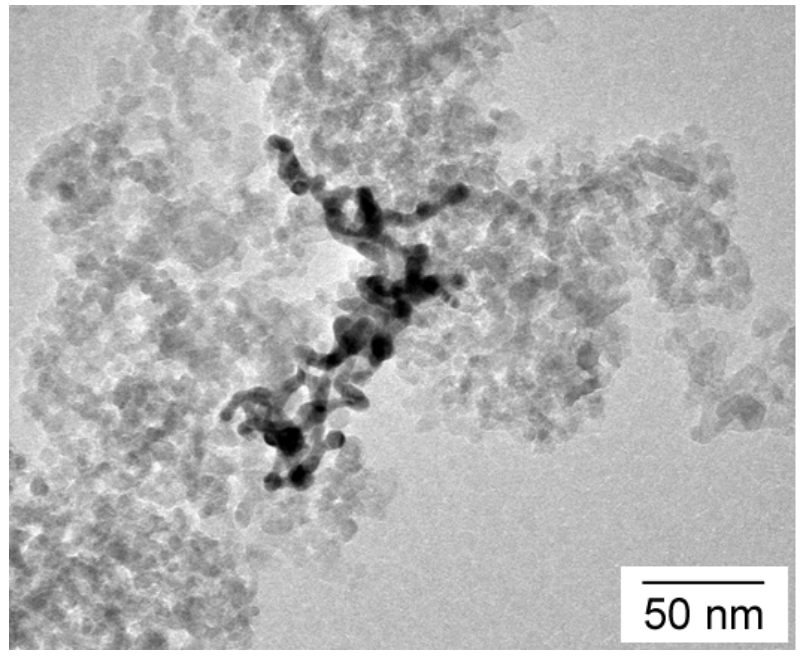

Figure S5. TEM image of $\mathrm{AuNW} / \gamma-\mathrm{Al}_{2} \mathrm{O}_{3}$ before water extraction.

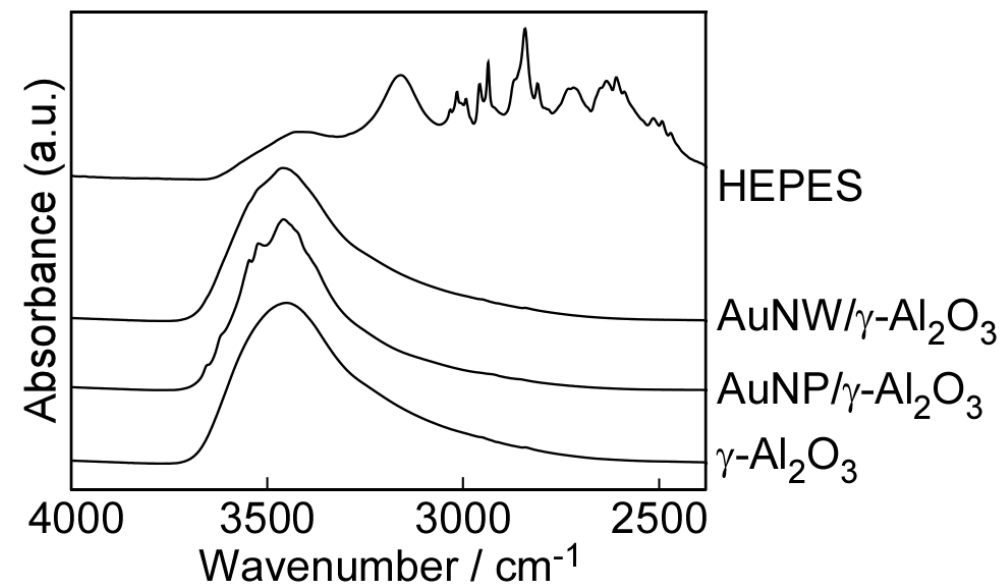

Figure S6. FT-IR spectra of HEPES, AuNW $/ \gamma-\mathrm{Al}_{2} \mathrm{O}_{3}, \mathrm{AuNP} / \gamma-\mathrm{Al}_{2} \mathrm{O}_{3}$, and $\gamma-\mathrm{Al}_{2} \mathrm{O}_{3}$. 
Table S1. Catalytic performance of $\gamma-\mathrm{Al}_{2} \mathrm{O}_{3}, \mathrm{AuNW} / \gamma-\mathrm{Al}_{2} \mathrm{O}_{3}$, and $\mathrm{AuNP} / \gamma-\mathrm{Al}_{2} \mathrm{O}_{3}$.

\begin{tabular}{lcc}
\hline & 1-PA conversion (\%) & AP yield (\%) \\
\hline$\gamma-\mathrm{Al}_{2} \mathrm{O}_{3}$ & 0 & 0 \\
$\begin{array}{l}\mathrm{AuNW} / \gamma-\mathrm{Al}_{2} \mathrm{O}_{3} \\
\text { after water extraction four times }\end{array}$ & 41 & 39 \\
$\begin{array}{l}\mathrm{AuNP} / \gamma-\mathrm{Al}_{2} \mathrm{O}_{3} \\
\text { after water extraction four times }\end{array}$ & 18 & 17 \\
\hline
\end{tabular}

1-PA: 1-phenylethyl alcohol, AP: acetophenone

Reaction conditions: 1-phenylethyl alcohol ( $30 \mu \mathrm{mol})$, catalyst (50 mg and $\mathrm{Au}=0.63 \mu \mathrm{mol}), \mathrm{K}_{2} \mathrm{CO}_{3}$ (0.1 g), air (1 atm), $40{ }^{\circ} \mathrm{C}$ and $2 \mathrm{~h}$.

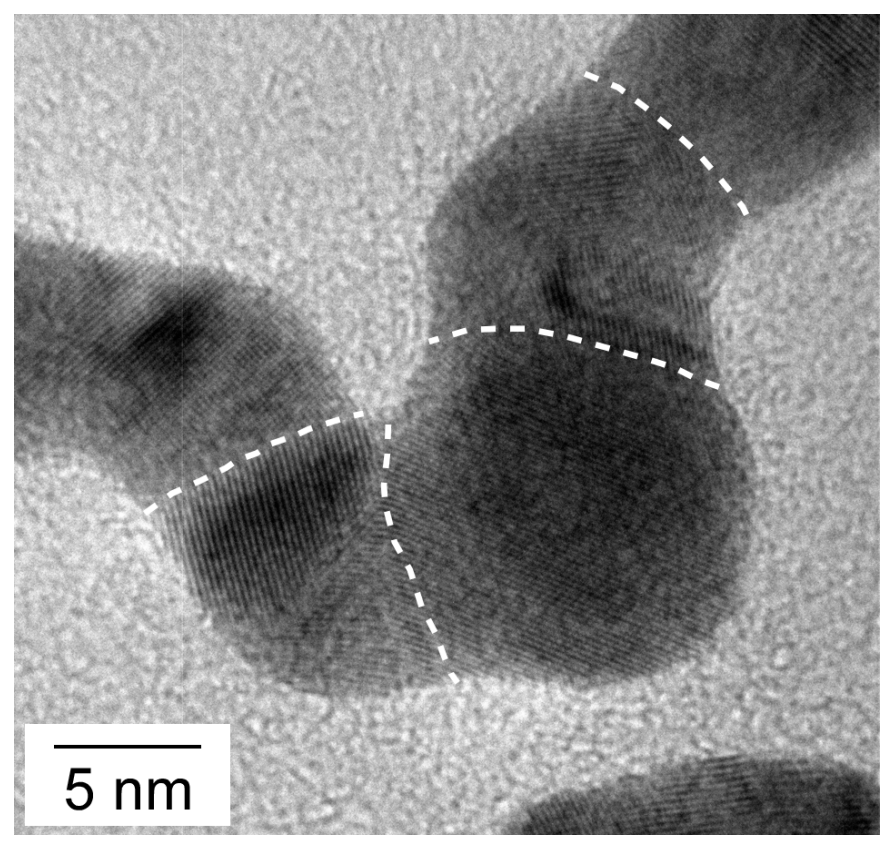

Figure S7. HR-TEM image of AuNW. 